\title{
Measurement of Defocus Level in Iris Images Using Different Convolution Kernel Methods
}

\author{
J. Miguel Colores-Vargas ${ }^{1}$, Mireya S. García-Vázquez ${ }^{1}$, \\ and Alejandro A. Ramírez-Acosta ${ }^{2}$ \\ ${ }^{1}$ Instituto Politécnico Nacional-CITEDI, Av. Del Parque No.1310, Tijuana BC, \\ \{colores, mgarciav\} @citedi.mx \\ ${ }^{2}$ MIRAL R\&D, 1047 Palm Garden, Imperial Beach, 91932 USA \\ ramacos10@hotmail.com
}

\begin{abstract}
During the video and fixed image acquisition procedure of an automatic iris recognition system, it is essential to acquire focused iris images. If defocus iris images are acquired, the performance of the iris recognition is degraded, because iris images don't have enough feature information. Therefore it's important to adopt the image quality evaluation method before the image processing. In this paper, it is analyzed and compared four representative quality assessment methods on the MBGC iris database. Through methods, it can fast grade the images and pick out the high quality iris images from the video sequence captured by real-time iris recognition camera. The experimental results of the four methods according to the receiver operating characteristic (ROC) curve are shown. Then the optimal method of quality evaluation that allows better performance in an automatic iris recognition system is founded. This paper also presents an analysis in terms of computation speed of the four methods.
\end{abstract}

Keywords: Convolution kernel, defocus, iris, quality, video.

\section{Introduction}

Nowadays, the development of better image quality metrics is an active area of research. The image quality plays a crucial role in the pattern matching system, particularly in automated biometric systems, like iris recognition where performance is based upon matching fine texture information in the annular region between the pupil and the sclera. Some studies report that using a high quality image affects recognition accuracy and can improve system performance [1]. Then, it is necessary to select a suitable image with high quality from an input sequence before all sequent operations. Otherwise, it can have a negative impact on segmentation algorithms and may be difficult to normalize and match, increasing the error probability [2], [3]. For example, in a capturing iris images system the subject to recognize usually moves his head in different ways gives rise to non-ideal images (with occlusion, off-angle, motion-blur and defocus) for recognition. A sample set of all these problems in images are shown in figure 1. As noted, choosing an appropriate image with quality seems a challenge. 

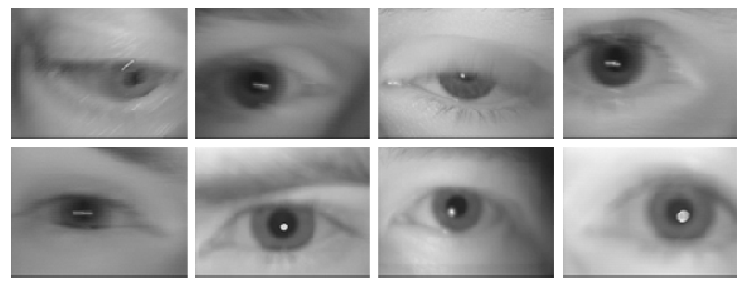

Fig. 1. Video sequences depicting various problems during acquisition of eyes images

To address the problem of image quality, related work on this subject can be placed into two categories [4]: local and global analyses. Local methods try to classify each pixel of the iris providing additional information about each region of the iris texture. The major feature of these approaches is that the evaluation of iris image quality is reduced to the estimation of a single or a pair of factors, such as out-offocus blur, motion blur, and occlusion. Iris quality should not be limited to one or two quality factors [2-6]. Zhang and Salganicaff [7] examine the sharpness of the region between the pupil and the iris. Moreover, the majority of previously methods require involvement of traditional segmentation methods that are iterative and thus computationally expensive.

In this work are analyzed the mainly used global methods. These approaches are good for quickly eliminating very poor quality images. They are based on convolution kernel for measuring defocus level in iris images. The segmentation is not required because the operator is applied to the entire image, giving the possibility of its implementation at hardware level.

This paper is organized as follows. Section 2 explains the principles of kernel-based defocus measurements and presents the main representative kernels used for of iris recognition systems. Methodologies for comparing kernels and results are given in Section 3, and Section 4 gives the conclusion.

\section{Measurement of Blurring and Defocus in Iris Images}

A clear image has relatively uniform frequency distribution in the 2D Fourier spectrum. On the other hand, the energy of a defocused or blurred image concentrates on the lower frequency part [7]. Therefore, spectral analysis of the frequency suggests that an effective way to estimate the degree of focus is measure its energy total at higher spatial frequencies. This is a common method in research on image quality assessment [8-11]. In a recognition system if an image can pass a minimum focus criterion, it will be used for recognition.

Thus, it needs a discrete formulation to obtain only the high frequency power, this can be solved filtering the low frequency part of the image, calculating the energy of the processing image (low frequency filtrated image) and set predefined threshold i.e. that images with energy values higher than the threshold are identified as clear images. Section 3 explains the methodology for calculating the thresholds, the optimum values for different filters are shown in table 1. 


\subsection{Convolution Kernels}

In order to obtain the high frequency power of the image, a proper high-pass convolution kernel is really important. In this section, it will give a brief description of convolution kernels presented in literature to determine the defocus degree in eyeiris images.

\subsubsection{Daugman's Convolution Kernel}

In his pioneering work [8], Daugman proved that the defocus primarily attenuates high spatial frequencies. Due to this relationship he improved the convolution operation in real-time, id est., to reduce the computational complexity of the Fourier transform proposed a high pass $8 \times 8$ convolution kernel to extract the high frequency of an image. The convolution kernel and his spectrum response are shown in figure 2 . The weights consists of two square box functions, one of size $8 \times 8$ with amplitude -1 , and the other one of size $4 \times 4$ and amplitude of +4 .

\begin{tabular}{|c|c|c|c|c|c|c|c|}
\hline-1 & -1 & -1 & -1 & -1 & -1 & -1 & -1 \\
\hline-1 & -1 & -1 & -1 & -1 & -1 & -1 & -1 \\
\hline-1 & -1 & 3 & 3 & 3 & 3 & -1 & -1 \\
\hline-1 & -1 & 3 & 3 & 3 & 3 & -1 & -1 \\
\hline-1 & -1 & 3 & 3 & 3 & 3 & -1 & -1 \\
\hline-1 & -1 & 3 & 3 & 3 & 3 & -1 & -1 \\
\hline-1 & -1 & -1 & -1 & -1 & -1 & -1 & -1 \\
\hline-1 & -1 & -1 & -1 & -1 & -1 & -1 & -1 \\
\hline
\end{tabular}

a)

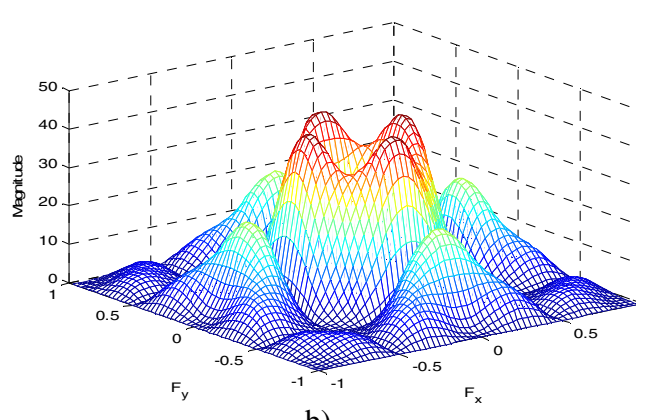

b)

Fig. 2. a) The $8 \times 8$ Convolution kernel proposed by Daugman [8] b) The frequency response (Fourier Spectrum)

\subsubsection{The Convolution Kernel of Wei et al.}

Wei et al. [9] also suggest a convolution kernel, with a similar shape as Daugman's for detecting defocused on still and segmented images. Additionally, they detect other problems presented in iris images; motion blur and occlusion. Each problem has its own peculiarity, so, the three features are used to classify them using SVM (Support Vector Machine) that is a machine learning algorithm method used for classification [11]. The total quality of an image according with their method is a vector Q (q1, q2, q3), where the values represent the levels from defocus, motion blur and occlusion respectively.

To determine the defocus degree they proposed a $5 \times 5$ convolution kernel as shown in figure 3. Compared with Daugman's $8 \times 8$ convolution kernel is also a lower frequencies filter but computationally less demanding. The operator is formed by three box functions, one of size $5 \times 5$ with amplitude -1 , one of size $3 \times 3$ with amplitude +3 , and the last one of size $1 \times 1$ with amplitude -2 . 


\begin{tabular}{|c|c|c|c|c|}
\hline-1 & -1 & -1 & -1 & -1 \\
\hline-1 & 2 & 2 & 2 & -1 \\
\hline-1 & 2 & 0 & 2 & -1 \\
\hline-1 & 2 & 2 & 2 & -1 \\
\hline-1 & -1 & -1 & -1 & -1 \\
\hline
\end{tabular}

a)

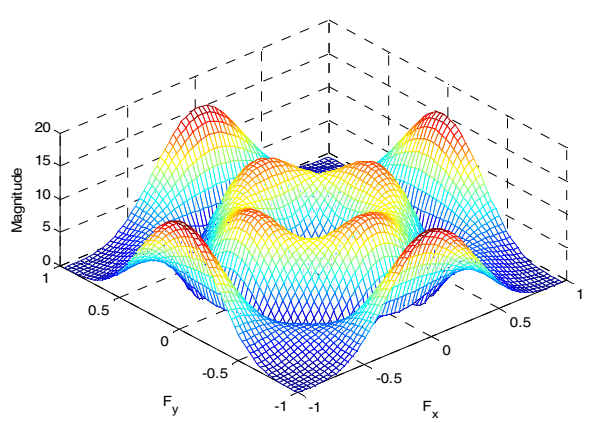

b)

Fig. 3. a) The $5 \times 5$ Convolution kernel proposed by Wei et al. b) The frequency response (Fourier Spectrum)

\subsubsection{Laplacian of Gaussian Convolution Kernel}

J. Wang et al. [10] propose a convolution kernel operator is based on a Laplacian of Gaussian function (LoG). The Laplacian give a 2-D isotropic measure of the second spatial derivative of an image, in the image highlights regions represent the rapid intensity change and is used for edge detection. The Gaussian smooth filter is applied first to the image; the purpose is to reduce the noise sensibility of the second derivative. They calls filter Laplacian of Gaussian filter. The combined filter function is centered on zero and with Gaussian standard deviation $\sigma$ has the form given by the equation:

$$
\operatorname{LoG}(x, y)=-\frac{1}{\pi \sigma^{4}}\left[1-\frac{x^{2}+y^{2}}{2 \sigma^{2}}\right] e^{-\frac{x^{2}+y^{2}}{2 \sigma^{2}}}
$$

Since the image is represented as a set of discrete pixels, they sought a discrete convolution kernel that can approximate the Laplacian operator. Set different values of the Gaussian, finally they used $\sigma=1.4$, this process results in a LoG operator as shown in figure 4.

\begin{tabular}{|c|c|c|c|c|c|c|c|c|}
\hline 0 & 1 & 1 & 2 & 2 & 2 & 1 & 1 & 0 \\
\hline 1 & 2 & 4 & 5 & 5 & 5 & 4 & 2 & 1 \\
\hline 1 & 2 & 4 & 5 & 5 & 5 & 4 & 2 & 1 \\
\hline 2 & 5 & 3 & -12 & -24 & -12 & 3 & 5 & 2 \\
\hline 2 & 5 & 0 & -24 & -40 & -24 & 0 & 5 & 2 \\
\hline 2 & 5 & 3 & -12 & -24 & -12 & 3 & 5 & 2 \\
\hline 1 & 2 & 4 & 5 & 5 & 5 & 4 & 2 & 1 \\
\hline 1 & 2 & 4 & 5 & 5 & 5 & 4 & 2 & 1 \\
\hline 0 & 1 & 1 & 2 & 2 & 2 & 1 & 1 & 0 \\
\hline
\end{tabular}

a)

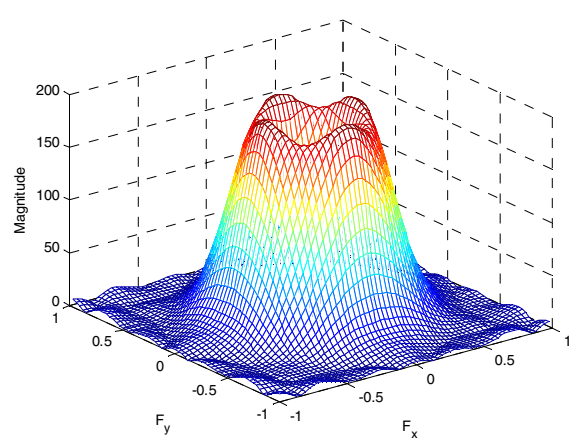

b)

Fig. 4. a) The 9x9 Convolution kernel based in Laplacian and Gaussian filter b) The frequency response (Fourier Spectrum) 


\subsubsection{The Convolution Kernel of Kang and Park}

Kang \& Park [11] propose 5x5 pixels sized convolution kernel as shown in figure 5. It consists of three square box functions, one of size $5 \times 5$ with amplitude -1 , one of size $3 \times 3$ and amplitude +5 , and other of size $1 \times 1$ and amplitude -5 .

However, they argue that their $5 \times 5$ pixels convolution kernel contains more high frequency bands than the 8x8 pixels convolution kernel proposed by Daugman [8]. From that, theoretically the operator can detect much better the high frequency of iris texture, using less processing time due to the short sized kernel.

\begin{tabular}{|c|c|c|c|c|}
\hline-1 & -1 & -1 & -1 & -1 \\
\hline-1 & -1 & 4 & -1 & -1 \\
\hline-1 & 4 & 4 & 4 & -1 \\
\hline-1 & -1 & 4 & -1 & -1 \\
\hline-1 & -1 & -1 & -1 & -1 \\
\hline
\end{tabular}

a)

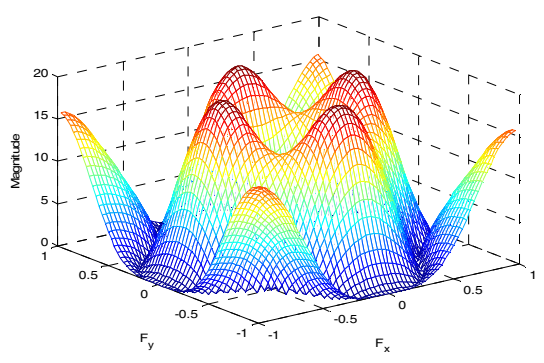

b)

Fig. 5. a) The $5 x 5$ Convolution kernel proposed by Kang \& Park for defocus measure b) The frequency response (Fourier Spectrum)

\section{Experimental Results}

\subsection{The Iris Video Dataset}

There are many iris databases such as the CASIA, UBIRIS and NIST ICE etc [15-17], but they do not include many motion blurred and optical defocus iris images. For the purpose of validating the effectiveness and compare the performance of the kernels in this work, it is produced a database with 4432 images for testing. The images were extracted from 100 different videos from the MBGC (Multiple Biometrics Grand Challenge) database.

The MBGC dataset used for this experiment was collected by The Computer Vision Research Lab at the University of Notre Dame and provided for the (MBGC) evaluation [13]. Actually this dataset has been distributed to over 100 research groups around the world. All videos were acquired using an LG2200 EOU iris capture system [14]. The camera uses near-infrared illumination of the eye. During acquisition, the eye is illuminated by one of three infrared LEDs: one above the lens, one to the left, and one to the right. The iris video sequences were digitized from the NTSC (National Television System Committee) video signal from the sensor. The signal produced was digitized by a DayStar XLR8 USB video digitizer attached to a Macintosh host system and stored in MPEG-4 format with a high bit rate allowance thus yielding with lossless encoding. The size for each frame in the video has 480 rows and 640 columns in 8 bits-grayscale space (intensity values between 0 to 255). Our dataset (4432 images ) contains 2077 clear images (positive samples) and 2355 
defocused images (negative samples). The entire collection of images was passed through the selection subjective process (based on human perception of quality). In images where we had uncertainty about whether it was a clear image or a defocus one, verification tests were implemented. Thus, the negative samples come from those images that cannot be segmented by Libor Masek recognition algorithm [18].

\subsection{Best Iris Image Selection}

For the testing process, it is computed the energy of every image from our iris database. The Receiver Operating Characteristic (ROC, also known as a Relative Operating Characteristic) curves was used to obtain the optimal threshold decision, we compared the difference between the two curves generated by energy values; one curve is generated by tests with defocused images and other curve by tests with focus images.

When it is considered the results of a particular test in two classes, it will rarely observe a perfect separation between the two groups. Indeed, the distribution of the test results will overlap. For every possible threshold point or criterion value you select to discriminate between the two classes. If an accepted/positive image (focus image) is a defocus image, it is called a false accept. The percentage of false accepts is called false accept rate (FAR). If a rejected image (defocus image) is a focus image, it is called a false reject. The percentage of false reject is called false reject rate (FRR).

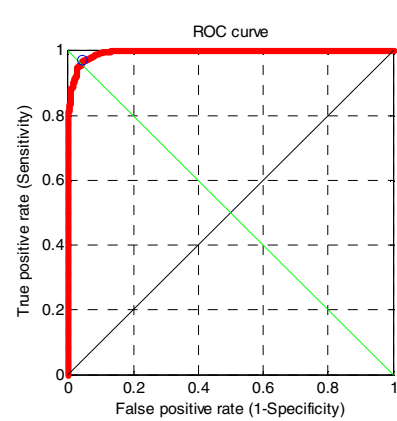

a)

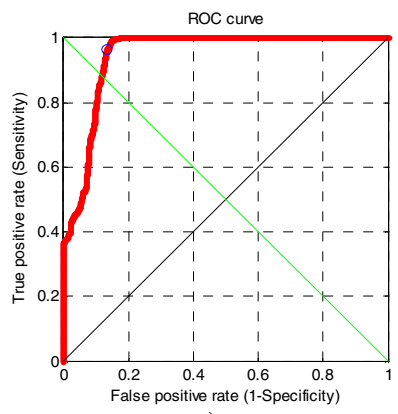

c)

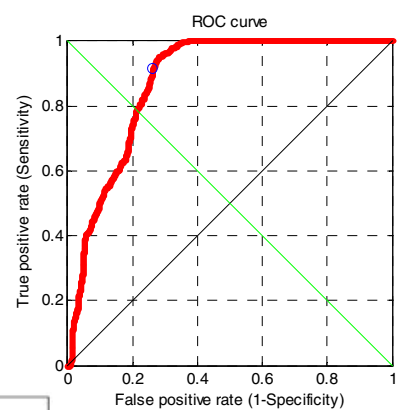

b)-

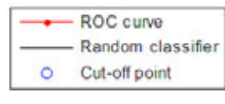

ROC curve

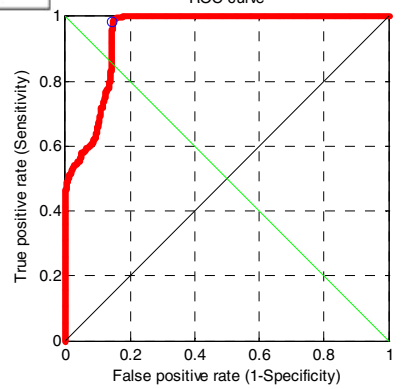

d)-

Fig. 6. ROC curves generated from the evaluated convolution kernels a) Daugman b) Wei et al c) LoG d) Kang \& Park 
In a ROC curve the true positive rate (Sensitivity) is plotted in function of the false positive rate (Specificity) for different cut-off points. Each point on the ROC plot represents a sensitivity/specificity pair corresponding to a particular decision threshold. Figure 6 not only indicates the performance of different methods but also provides information of how much the performance of a given method can vary. That is, the ROC curve is directly related to these two distance distributions. Therefore it is set the threshold for distinguishing the two kinds of images. By the threshold good quality images in the testing dataset passed the evaluation, and the poor quality images were rejected. The False Acceptance Rate (FAR) and False Rejection Rate (FRR) of all kernels are shown in the table 1.

Table 1. The table shows the parameters of the curves generated by experiments and the optimal thresholds desicion for each kernel

\begin{tabular}{|c|c|c|c|c|c|c|c|}
\hline \multirow[b]{2}{*}{ Kernel } & \multicolumn{2}{|c|}{ Clear iris images } & \multicolumn{2}{|c|}{ Defocus iris images } & \multirow{2}{*}{$\begin{array}{l}\text { Optimal } \\
\text { Decision } \\
\text { threshold }\end{array}$} & \multirow[b]{2}{*}{$\begin{array}{c}\text { FAR } \\
(\%)\end{array}$} & \multirow[b]{2}{*}{$\begin{array}{r}\text { FRR } \\
(\%)\end{array}$} \\
\hline & Mean & $\begin{array}{l}\text { Standard } \\
\text { deviation }\end{array}$ & Mean & $\begin{array}{l}\text { Standard } \\
\text { deviation }\end{array}$ & & & \\
\hline Daugman & 55.063 & 8.9134 & 26.838 & 5.7642 & 39.9490 & 2.8 & 3.6 \\
\hline Wei et al. & 19.928 & 4.5584 & 12.291 & 5.3759 & 14.1631 & 8.3 & 2.6 \\
\hline LoG & 108.49 & 10.651 & 72.88 & 14.658 & 92.0776 & 3.7 & 5.2 \\
\hline Kang \& Park & 25.792 & 6.0658 & 13.25 & 3.9636 & 15.8247 & 1.6 & 2.3 \\
\hline
\end{tabular}

The table contains the obtained results, the first column identifies the evaluated kernel, the next four columns contain the parameters of the distribution curve produced from the tests with defocus and clear images, the sixth column contains the optima's threshold for discrimination of defocus iris images, and the last columns are the error percentages for every kernel. As shown in table, the best performance scores (minimum FAR and FRR) was generated by the kernel proposed by Kang \& Park followed of the Daugman kernel who presented also low error rates. The worse results performance kernels were presented by kernels proposed in $[9,10]$, these kernels had the highest error rates.

To compare the convolution kernels in terms of speed is calculate the total multiplication count (TMC). With the $8 \times 8$ and 9x9 pixels convolution kernels, the convolution value is calculated per every fourth row and fourth column in the iris image [19] the TMC $=1228800(8 \times 8 \times 640 / 4 \times 480 / 4)$ and $\mathrm{TMC}=1555200(9 \times 9 \times 640 / 4 \times$ $480 / 4)$. For a $5 \times 5$ pixels convolution kernel, the convolution value is calculated per every third row and third column, the TMC $=852000(5 \times 5 \times 640 / 3 \times 480 / 3)$. The $5 \times 5$ pixels convolution kernels are $30.66 \%$ faster than the $8 \times 8$ pixels convolution kernels.

\section{Conclusions}

In this paper, it was analyzed four representative convolution kernels for image quality assessment on the MBGC iris database. They are a simple, fast quality descriptors based on the Fourier spectral of an iris image is defined to discriminate from any given eye image video-sequence, clear iris images from low quality images due to motion blur and defocus. The performance of every convolution kernel 
analyzing the relationship between the quality of iris images in terms of ROC curves. Some discussions can be given based on the results:

- The algorithm deals with the whole image, avoiding the location and segmentation of the pupil and iris.

- The execution time of the algorithm is suitable for a real-time recognition system.

- The algorithm is effective for the defocused and motion blurred images, but it is ineffective for the occluded images of eyelids and eyelashes there are rich in middle and high frequency components, which is an important factor in discriminating such images from clear images.

- It can adjust the threshold to get a satisfied FAR and a tradeoff between FAR and FRR.

From the above analysis and results is concluded that the Kang \& Park convolution kernel is superior to the other three kernels in terms of speed and accuracy.

Acknowlegment. This work was realized under grant SIP20100030.

\section{References}

1. Gamassi, M., Lazzaroni, M., Misino, M., Piuri, V.: Quality assessment of biometric systems: a comprehensive perspective based on accuracy and performance measurement. IEEE Transactions on Instrumentation and Measurement 54, 1489-1496 (2005)

2. Chen, Y., Dass, S.C., Jain, A.K.: Localized iris image quality using 2-D wavelets. In: Zhang, D., Jain, A.K. (eds.) ICB 2005. LNCS, vol. 3832, pp. 373-381. Springer, Heidelberg (2005)

3. Kalka, N.D., Zuo, J., Schmid, N.A., Cukic, B.: Image quality assessment for iris biometric. In: SPIE 6202: Biometric Technology for Human Identification III, vol. 6202, pp. D1-D11 (2006)

4. Zuo, J., Schmid, N.A.: Global and local quality measures for NIR iris video. In: IEEE Computer Society Conference on Computer Vision and Pattern Recognition Workshops, CVPRW 2009, pp. 120-125 (2009)

5. Zhu, X.-D., Liu, Y.-N., Ming, X.: A quality evaluation method of iris images sequence based on wavelet coefficients in region of interest. In: CIT '04: Proceedings of the The Fourth International Conference on Computer and Information Technology, Washington, DC, USA, pp. 24-27. IEEE Computer Society, Los Alamitos (2004)

6. Ma, L., Tan, T., Wang, Y.: Personal identification based on iris texture analysis. IEEE Transactions on Pattern Analysis and Machine Intelligence 25(12), 1519-1533 (2003)

7. Zhang, G., Salganicoff, M.: Method of measuring the focus of close-up image of eyes. Tech. Rep. 5953440, United States Patent (1999)

8. Daugman, J.G.: How iris recognition works. IEEE Trans. Circ. Syst. Video Tech. 14(1), 21-30 (2004)

9. Wei, Z., Tan, T., Sun, Z., Cui, J.: Robust and Fast Assessment of Iris Image Quality. In: Zhang, D., Jain, A.K. (eds.) ICB 2005. LNCS, vol. 3832, pp. 464-471. Springer, Heidelberg (2005) 
10. Wang, J., He, X., Shi, P.: An Iris Image Quality Assessment Method Based on Laplacian of Gaussian Operation. In: MVA2007 IAPR Conference on Machine Vision Applications, Tokyo, Japan, May 16-18, pp. 248-251 (2007)

11. Kang, B.J., Park, K.R.: A study on iris image restoration. In: International Conference on Audio- and Video-Based Biometric Person Authentication, pp. 31-40 (2005)

12. Burges, J.C.: A Tutorial on Support Vector Machines for Pattern Recognition. Data Mining and Knowledge Discovery 2, 121-167 (1998)

13. Multiple Biometric Grand Challenge, http: / / face.nist.gov/mbgc/

14. Jonathon Phillips, P., Bowyer, K.W., Flynn, P.J., Liu, X., Todd Scruggs, W.: The Iris Challenge Evaluation 2005. In: Biometrics: Theory, Applications and Systems, Washington, DC (September 2008)

15. CASIA iris image database, http://www. sinobiometrics.com

16. National Institute of Standards and Technology (NIST). Iris Challenge Evaluation (2008), http://iris.nist.gov/ice/

17. Proença, H., Alexandre, L.A.: UBIRIS: A noisy iris image database. In: Roli, F., Vitulano, S. (eds.) ICIAP 2005. LNCS, vol. 3617, pp. 970-977. Springer, Heidelberg (2005)

18. Masek, L.: Recognition of human iris patterns for biometric identification. Master's thesis, University of Western Australia (2003)

19. Daugman, J.G.: How Iris Recognition Works. IEEE Trans. on Circuits and Systems for Video Technology 14(1), 21-30 (2004) 